\title{
Relação entre a miocardite e a infecção causada pelo SARS-CoV-2
}

\author{
Relationship between myocarditis and infection caused by SARS-CoV-2 \\ Relación entre miocarditis e infección causada por SARS-CoV-2
}

Juliana Vanessa Cavalcante Souza ORCID: https://orcid.org/0000-0003-1455-4883 Laboratório Central de Alagoas, Brasil E-mail: ju.biomedica@hotmail.com Karene Ferreira Cavalcante ORCID: https://orcid.org/0000-0001-5411-7149 Laboratório Central do Ceará, Brasil E-mail:karene@edu.unifor.br

Denys Wanderson Pereira Frazão ORCID: https://orcid.org/0000-0002-6527-0912 IPR Cursos e Engenharia Clínica, Brasil

E-mail: denysfrazao@gmail.com

Alexandre Mendes Soares

ORCID: https://orcid.org/0000-0002-9291-0452 Centro Universitário UNINOVAFAPI, Brasil

E-mail: alexandresoares.bio@hotmail.com

Ana Carolina Hortencio Garcia

ORCID: https://orcid.org/0000-0001-5153-254X Centro Universitário de Brasília, Brasil E-mail: carolinahortencio@gmail.com

Danilo do Nascimento Viana

ORCID: https://orcid.org/0000-0002-0049-0383

Centro Universitário UNINOVAFAPI, Brasil

E-mail: danilonviana@gmail.com

Camila dos Santos Xavier

ORCID: https://orcid.org/0000-0002-6951-4793 Universidade Federal do Piauí, Brasil

E-mail: camiladsxavier@gmail.com

Denis Romulo Leite Furtado

ORCID: https://orcid.org/0000-0002-6266-1061

Laboratório Central do Piauí, Brasil

E-mail: kelly-rego@bol.com.br

Lizandro de Freitas Pessoa

ORCID: https://orcid.org/0000-0001-6941-6547

Centro Universitário UNIFACEMA, Brasil

E-mail: lizandrofreitas23@hotmail.com

Márcia Laís Fortes Rodrigues Mattos

ORCID: https://orcid.org/0000-0002-5202-5010

Faculdade de Tecnologia de Teresina, Brasil E-mail: marcialaismattos@gmail.com

Manoel Lopes da Silva Filho

ORCID: https://orcid.org/0000-0002-1753-1057 Universidade Federal do Piauí, Brasil

E-mail: manoellopes@ufpi.edu.br

Manuelle Rodrigues da Silva

ORCID: https://orcid.org/0000-0003-2544-7047

Faculdade de Tecnologia de Teresina, Brasil

E-mail: manursd@outlook.com

Kelly Maria Rêgo Da Silva

ORCID: https://orcid.org/0000-0003-3070-4060

Laboratório Central do Piauí, Brasil

E-mail: kellyma.aria@gmail.com 


\title{
Resumo
}

Com base em dados da China, onde a pandemia se deu origem, a lesão cardíaca se tornou uma característica proeminente da doença, acometendo até $30 \%$ dos pacientes e auxiliando para $40 \%$ das mortes. Foram descritas diversas complicações cardiovasculares, como lesão miocárdica (20\% dos casos), arritmias (16\%), miocardite (10\%), além de insuficiência cardíaca (IC) e choque (até 5\% dos casos). O objetivo desse trabalho é informar e descrever sobre a nova miocardite ocasionada pelo novo vírus. E demonstrar a importância para novos estudos relacionados com a nova doença. Foram utilizadas todas as publicações que possuírem dados sobre a nova miocardite em pacientes com o novo SARS-CoV-2, publicações científicas, oficiais da Organização Mundial da Saúde e governamentais, dentre a determinada data (2019-2021). Foram encontrados aproximadamente 500 artigos, foram verificados os idiomas, e se realmente se tratava sobre o tema da nova miocardite relacionada ao novo vírus, então foram obtidos 39 artigos para a realização da discussão. A lesão do miocárdio, sinalizada por marcadores foi evidenciada já nos primeiros casos da doença. O Conselho Nacional de Saúde da China demonstrou em um relatório, 12\% dos pacientes sem problemas cardiovasculares. Sendo assim, já que o SARS-CoV-1 e SARS-CoV-2 atinge as células através da enzima conversora de angiotensina 2, onde isso possivelmente acontece também em pacientes infectados com o novo vírus. Porém ainda são necessárias mais pesquisas para comprovação dessas possíveis teorias.

Palavras-chave: Miocardite; Saúde; Novo coronavírus.

\begin{abstract}
Based on data from China, where the pandemic originated, cardiac injury has become a prominent feature of the disease, affecting up to $30 \%$ of patients and helping $40 \%$ of deaths. Several cardiovascular complications have been described, such as myocardial injury (20\% of cases), arrhythmias (16\%), myocarditis (10\%), in addition to heart failure (HF) and shock (up to 5\% of cases). The aim of this work is to inform and describe the new myocarditis caused by the new virus. And demonstrate the importance for further studies related to the new disease. All publications that have data on the new myocarditis in patients with the new SARS-CoV-2, scientific publications, official from the World Health Organization and government, between a certain date (2019-2021) were used. Approximately 500 articles were found, languages were checked, and if it was really about the theme of the new myocarditis related to the new virus, then 39 articles were obtained for the discussion. Myocardial injury, signaled by markers, was evidenced in the first cases of the disease. The National Health Council of China demonstrated in a report, $12 \%$ of patients without cardiovascular problems. Thus, since SARS-CoV-1 and SARS-CoV-2 reach cells through the angiotensin-converting enzyme 2, where this possibly also happens in patients infected with the new virus. However, more research is still needed to prove these possible theories.
\end{abstract}

Keywords: Myocarditis; Health; New coronavirus.

\section{Resumen}

Según datos de China, donde se originó la pandemia, la lesión cardíaca se ha convertido en una característica destacada de la enfermedad, que afecta hasta al 30\% de los pacientes y ayuda al $40 \%$ de las muertes. Se han descrito varias complicaciones cardiovasculares, como lesión miocárdica (20\% de los casos), arritmias (16\%), miocarditis (10\%), además de insuficiencia cardíaca (IC) y shock (hasta un 5\% de los casos). El objetivo de este trabajo es informar y describir la nueva miocarditis provocada por el nuevo virus. Y demostrar la importancia de realizar más estudios relacionados con la nueva enfermedad. Se utilizaron todas las publicaciones que tienen datos sobre la nueva miocarditis en pacientes con el nuevo SARS-CoV-2, publicaciones científicas, oficiales de la Organización Mundial de la Salud y del gobierno, entre una fecha determinada (2019-2021). Se encontraron aproximadamente 500 artículos, se revisaron los idiomas y si realmente se trataba del tema de la nueva miocarditis relacionada con el nuevo virus, entonces se obtuvieron 39 artículos para la discusión. La lesión miocárdica, señalada por marcadores, se evidenció en los primeros casos de la enfermedad. El Consejo Nacional de Salud de China mostró en un informe, el 12\% de los pacientes sin problemas cardiovasculares. Por lo tanto, dado que el SARS-CoV-1 y el SARS-CoV-2 llegan a las células a través de la enzima convertidora de angiotensina 2, esto posiblemente también ocurra en pacientes infectados con el nuevo virus. Sin embargo, aún se necesita más investigación para probar estas posibles teorías.

Palabras clave: Miocarditis; Salud; Nuevo coronavirus.

\section{Introdução}

O equilíbrio do sistema cardiovascular é realizado por eficazes mecanismos, que regulam a frequência cardíaca e a pressão arterial. Várias patologias (fatores de risco) como diabetes, obesidade, hipertensão, idade, dentre outros podem facilitar a miocardite (Fang, 2020).

Vários fatores contribuíram para os fatores de risco como a mudança de rotina, de empregos, de estudos, alimentação, redução de exercícios físicos, dentre outros. Outros fatores como o tabagismo e controle estresse psicoemocional (Kim, 2020).

O padrão de comportamento e os hábitos mudaram condições de oferta e demanda, consumo, modismo e outras 
modificações, aumentaram o risco de diabetes ou colesterol, dentre outras alterações (Million, 2020).

Com base em dados da China, onde a pandemia se deu origem, a lesão cardíaca se tornou uma característica proeminente da doença, acometendo até 30\% dos pacientes e auxiliando para $40 \%$ das mortes. Foram descritas diversas complicações cardiovasculares, como lesão miocárdica (20\% dos casos), arritmias (16\%), miocardite (10\%), além de insuficiência cardíaca (IC) e choque (até 5\% dos casos) (Zheng, 2020).

O objetivo desse trabalho é informar e descrever sobre a nova miocardite ocasionada pelo novo vírus. E demonstrar a importância para novos estudos relacionados com a nova doença.

\section{Metodologia}

Trata-se de uma pesquisa documental, de objetivo descritivo e explicativo com abordagem qualitativa e quantitativa. Essa pesquisa busca através de publicações cientificas descrever seus resultados, explicando suas causas e efeitos (KAUARK, 2010).

Foram utilizadas todas as publicações que possuírem dados sobre a nova miocardite em pacientes com o novo SARSCoV-2, publicações cientificas, oficiais da Organização Mundial da Saúde (World Health Organization - WHO) e governamentais, dentre a determinada data (2019-2021).

Os dados foram coletados utilizando as bases cientificas: Literatura Latino-Americana e do Caribe em Ciências da Saúde (LILACS), National Library of medicine (PUBMED), PERIODICOS, SCIENCE DIRECT, Biblioteca Virtual em saúde - (BVS), Scientific Eletronic Library Online (SCIELO), Cochrane Library, HighWire Press, Scopus e Elsevier.

Foram analisados os trabalhos a nível mundial, nacional e estadual; e comparados para maior relevância dos resultados. Enaltecendo os resultados trabalhados.

Foram utilizados nessa pesquisa todos os trabalhos que relatam sobre a nova miocardite em pacientes com o novo coronavírus, publicados nos anos de 2019 a 2021, nos idiomas: inglês, espanhol e português. foram excluídos todos os demais trabalhos que não se tratarem do novo coronavírus, e publicações em outros idiomas não citados a cima.

\section{Resultados e Discussão}

Foram encontrados aproximadamente 500 artigos, foram verificados os idiomas, e se realmente se tratava sobre o tema da nova miocardite relacionada ao novo vírus, então foram obtidos 39 artigos para a realização da discussão.

A lesão do miocárdio, sinalizada por marcadores foi evidenciada já nos primeiros casos da doença. O Conselho Nacional de Saúde da China demonstrou em um relatório, 12\% dos pacientes sem problemas cardiovasculares (Guan, 2020).

\subsection{Mecanismo de ataque celular do SARS-CoV-2}

A infecção por SARS-CoV-2 se inicia pela proteína spike da superfície do vírus ao receptor da Enzima Conversora de Angiotensina II humana após a ativação da proteína spike pela protease serina 2 transmembranas (TMPRSS2) (Hoffmann, 2020).

A (ECA-2) ela é ativada no pulmão, principalmente nos alvéolos do tipo II, que funciona com porta de entrada. Após essa ligação o vírus forma downregulation, o que determina a crescimento dos níveis de angiotensina II, o que pode deletar a ativação do sistema renina-angiotensina-aldosterona, causando diversos efeitos como por exemplo vaso constrição, alteração de permeabilidade vascular, remodelamento miocárdico e injúria pulmonar aguda, o que ocasiona a maioria dos sintomas pulmonares na doença (Zhao, 2020).

Como podemos observar na Figura 1, o vírus do COVID-19 liga-se por meio da proteína spike da superfície viral ao receptor da Enzima Conversora de Angiotensina II: 
Figura 1 - O SARS-CoV-2 liga-se por meio da proteína spike da superfície viral ao receptor da ECA-2 humana após a ativação da proteína spike pela TMPRSS2. SARS-CoV: Coronavírus da síndrome respiratória aguda grave; SARS-COV-2: Coronavírus da síndrome respiratória aguda grave 2; COVID-19: doença do Coronavírus 2019; ECA-2: enzima conversora de angiotensina-2; TMPRSS2: serina protease transmembrana-2.

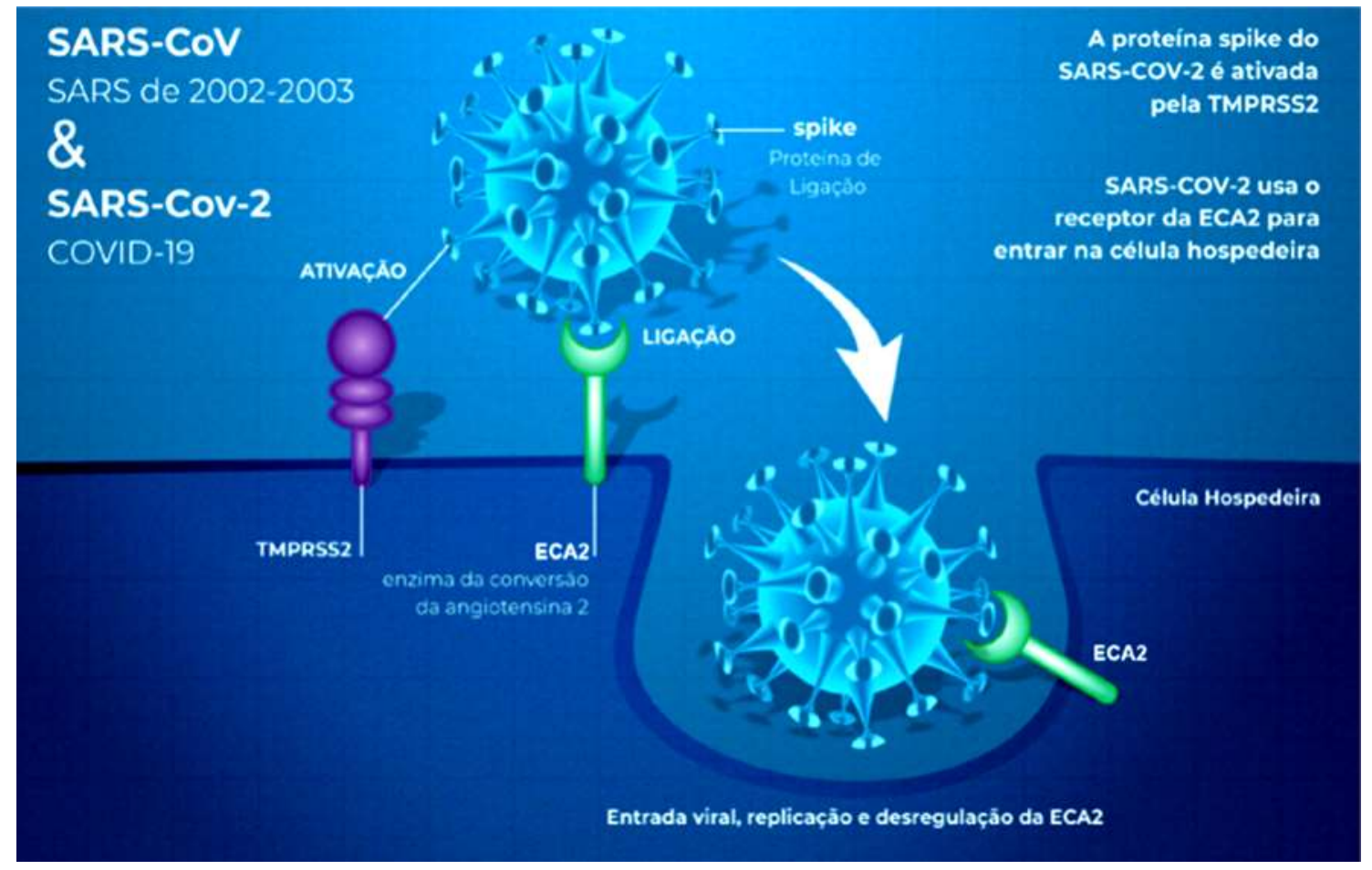

Fonte: Costa, et al. (2020).

A ECA-2 ela neutraliza os efeitos da angiotensina II quando ela se encontra em maior quantidade no sistema reninaangiotensina, ela também se encontra em grande quantidade no coração. Quando a angiotensina II se encontra em grande quantidade ela causa hipertensão arterial sistêmica, insuficiência cardíaca e aterosclerose; por isso se há uma necessidade na transformação da angiotensina II em angiotensina I para gerar um efeito de proteção no coração (Turner, 2020).

Essa enzima também funciona para o coração, pulmão, intestino, rins e endotélio vascular, fornecendo um mecanismo para a disfunção de múltiplos órgãos que pode ser observada na infecção pelo vírus do COVID-19 (Qiao, 2020).

\subsection{Nova lesão miocárdica ocasionada pelo Novo Coronavírus}

O aumento da troponina nos hospitais demonstrou sua ligação com a grande mortalidade dos pacientes com o Novo Coronavírus (Zheng, 2020).

Estudos realizados na cidade de Wuhan, realizado com 416 pacientes, foi demonstrado que a prevalência era nos idosos, não havendo discriminação de sexo. O fator de risco mais frequente foi a hipertensão arterial (30,5\%), injúria miocárdica (19,7\%), sem hipertensão (59\% x 23\%), doença arterial coronária (DAC) (29,3\% x 6,0\%); doença cerebrovascular $(15,9 \% \times 2,7 \%)$ e insuficiência cardíaca $(14,6 \%$ x 1,5\%) (p < 0,001 para todas as variáveis) (Patel, 2020).

Outro trabalho utilizado mostra uma coorte retrospectiva unicêntrica que avaliou 187 pacientes com Coronavírus. Também afetou mais os idosos, onde 35\% se tratava de pacientes com algum fator de risco (cardiomiopatia ou hipertensão). Sendo que $23 \%$ evoluíram para óbito. Os pesquisadores notaram um crescimento do nível de troponina nos pacientes em $27,8 \%$ dos casos. Os pacientes que tiveram o nível baixo de troponina não apresentaram mortalidade. Onde o crescimento da troponina variou de 15 a 20 dias até o óbito do paciente (Zheng, 2020). 
Juntamente com a análise de troponina também se observou a elevação de outros marcadores como: ferritina, dímero D, interleucina-6 e desidrogenase lactato, o que pode ter levado a uma lesão miocárdica (VADUGANATHAN, 2020).

\subsection{Mecanismos da nova lesão miocárdica causada pelo Novo Coronavírus}

O mecanismo em si ainda não foi descrito, porém existem teorias, como o aumento do estresse cardíaco devido a insuficiência respiratória e hipoxemia, síndrome coronariana aguda (SCA), lesão indireta da resposta inflamatória sistêmica, infecção miocárdica direta pelo vírus do COVID-19, dentre outros (Fang, 2020).

\subsection{Lesão miocárdica ligada ao desequilíbrio entre oferta e demanda de oxigênio}

As situações de grande estresse como sepse ou outros problemas em pacientes com o novo vírus, estão ligados ao aumento dos biomarcadores, complicando alguns pacientes. $\mathrm{O}$ acontecimento mais esperado é uma alteração na quantidade de oxigênio, sem ruptura da placa ateromatosa pode ocasionar o infarto do miocárdio tipo II (Wan, 2020).

\subsection{Lesão microvascular}

A maioria das lesões que ocorrem no miocárdio ocorre por formação de microtrombos nas vasculaturas, com a alteração de hipercoagulabilidade igual a coagulação intravascular disseminada. Essas alterações são facilmente encontradas em pacientes com o novo vírus, principalmente os que foram a óbito (BRASIL, 2020).

Esses mecanismos da coagulação intravascular disseminada, no caso de infecção generalizada e síndrome do desconforto respiratório agudo, ocorre uma exaustão de alguns sistemas como o de coagulação e fibrinolítico facilitando sangramentos e trombose. Também ocorre um aumento das citocinas inflamatórias como a IL-6, também o fator de necrose tumoral-alfa, lesões endoteliais, aumentando a expressão do fator tecidual o que decidindo um estado pró-trombótico (Million, 2020).

Com isso a presença de inflamação e ativação imune que respondem a infecção grave pelo novo vírus, podem decidir a lesão miocárdica e disfunção microvascular (KIM, 2020).

\subsection{Resposta inflamatória}

A resposta inflamatória sistêmica é o mecanismo que tem relação a lesão cardíaca. Pesquisas mostram que níveis altos de marcadores como: citocinas, incluindo IL-6, proteína C-reativa, TNF- $\alpha$, interleucina-2R (IL-2R) e ferritina estão associados ao caos levando a óbito (SARAYANI, 2020).

Pesquisas mostram também que o óxido nítrico possa ser o responsável pela depressão do miocárdio em estados de intensa inflamação (COLSON, 2020).

\subsection{Síndrome coronariana}

O Novo Coronavírus pode apresentar sinais e sintomas da Síndrome coronariana aguda não obstrutiva como alterações eletrocardiográficas sugestivas de isquemia miocárdica ou infarto agudo do miocárdio e dor toráxica, complicando o seu diagnostico (PEARSON, 2020).

Atualmente os trabalhos publicados até agora não mostram Síndrome coronariana aguda não obstrutiva por quebra da placa epicárdica, como mecanismo para a lesão cardíaca nessa patologia. Porém existem teorias que demonstram a associação entre infecção de um grande risco dessa complicação; a disfunção endotelial proveniente da infecção e inflamação pode determinar vasoconstricção, com redução do fluxo coronariano (CARNELOSSO, 2020). 


\subsection{Lesão no miocárdio}

Os casos de miocardites na nova doença mostram confirmações de inflamações cardíacas, porém não determinaram o seu mecanismo. Uma possível teoria do mecanismo seria uma infecção viral direta no coração, resultando uma miocardite (ALVES, 2006).

Existem poucos relatos registrados dessa patologia, que foram confirmadas por biópsia com inclusões virais ou RNA viral detectado no tecido do miocárdio. Entretanto não havia a presença do vírus nos cardiomiócitos, somente nos macrófagos no interstício cardíaco. Uma teoria dessa lesão seria através de uma vasculite mediada por infecção, pois existem pesquisas que mostram vasculite com infiltração de monócitos e linfócitos e lesão de células do coração (ARAUJO, 2007).

A entrada direta do vírus nas células pode gerar essa vasculite ou a própria resposta imunológica levando a um aumento da sensibilidade (MARÃES et al. 2005).

\section{Conclusão}

Esse trabalho conclui que de acordo com as pesquisas se torna claro uma relação entre a presença de lesão miocárdica, identificado pelo aumento da troponina relacionado a uma piora de pacientes com o novo vírus. Tudo isso ocorre relacionado também com o aumento dos marcadores confirmando a clínica de miocardite.

Sendo assim, já que o SARS-CoV-1 e SARS-CoV-2 atinge as células através da ECA-2, onde isso possivelmente acontece também em pacientes infectados com o novo vírus. Porém ainda são necessárias mais pesquisas para comprovação dessas possíveis teorias.

\section{Referências}

Alves, V. S., \& Nunes, M. O. (2006). Educação em saúde na atenção médica ao paciente com hipertensão arterial no Programa Saúde da Família. Interface Comum. Saúde Educ; 10(19):131-147.

Araújo, J. C., Guimarães, A. C. (2007). Controle da Hipertensão. Arterial em uma Unidade de Saúde da Família. Rev. Saúde Pública; 41(3):368-374.

Brasil. (2020). Segundo Posicionamento do Departamento de Hipertensão Arterial da Sociedade Brasileira de Cardiologia (DHA/SBC) sobre inibidores da enzima de conversão da angiotensina (IECA), bloqueadores dos receptores da angiotensina (BRA) e Coronavírus (COVID-19).

Carnelosso, M. L. C. (2008). Iniciativa Carment: resultados e tendências das prevalências dos fatores de risco cardiovasculares [tese]. Goiânia (GO): Programa Multi-institucional de Pós-Graduação em Ciências da Saúde, Convênio Centro Oeste/Universidade de Brasília (UNB), Universidade Federal de Goiás (UFG), Universidade Federal de Mato Grosso do Sul (UFMS).

Costa, I. B. S. S., Bittar, C. S., Rizk, S. I., Araújo Filho, A. E, Santos, K. A. Q., Machado, T. I. V., et al. (2020). The heart and COVID-19: what cardiologists need to know. Arq Bras Cardiol.

Colson, P., Rolain, J. M., \& Raoult, D. (2020). Chloroquine for the 2019 novel coronavirus SARS-CoV-2. International Journal of Antimicrobial Agents; $55(3)$.

Fang, L., Karakiulakis, G., \& Roth, M. (2020). Are patients with hypertension and diabetes mellitus at increased risk for COVID-19 infection? Lancet Respir Med; 8(21).

Guan, W. J., Liang, W. H., Zhao, Y., et al. (2020). China Medical Treatment Expert Group for Covid-19. Comorbidity and its impact on 1590 patients with Covid-19 in China: A Nationwide Analysis. Eur Respir J.

Hoffmann, M., Kleine-Weber, H., Schroeder, S., Krüger, N., Herrler T., Erichsen, S., et al. (2020). SARS- CoV-2 cell entry depends on ACE2 and TMPRSS2 and is blocked by a clinically proven protease inhibitor. Cell; 181(2):271-80.

Kauark, F. (2010). Metodologia da pesquisa: guia prático / Fabiana Kauark, Fernanda Castro Manhães e Carlos Henrique Medeiros. - Ita- buna: Via Litterarum editora, $88 \mathrm{p}$.

Kim, Y., Kwon, O., Paek, J. H., et al. (2020). Two distinct cases with COVID-19 in kidney transplant recipients. American journal of transplantatio: official journal of the American Society of Transplantation and the American Society of Transplant Surgeons.

Marães, V. R., Silva, E., Catai, A. M., Novais, L. D., Moura, M. S., Oliveira, L., et al. (2005). Identification of anaerobic threshold using heart rate response during dynamic exercise. Braz J Med Biol Res; 38(5):731-5.

Million, M. (2020). Early treatment of COVID-19 patients with hydroxychloroquine and azithromycin: A retrospective analysis of 1061 cases in Marseille, France. Travel medicine and infectious disease. 
Research, Society and Development, v. 10, n. 7, e20710716357, 2021

(CC BY 4.0) | ISSN 2525-3409 | DOI: http://dx.doi.org/10.33448/rsd-v10i7.16357

Patel, A. B., \& Verma, A. (2020). COVID-19 and Angiotensin-Converting Enzyme Inhibitors and Angioרtensin Receptor Blockers: What Is the Evidence? JAMA.

Pearson, T. A., Walls, S., \& Lewis, C. (2001). Dissecting the "black box" of community intervention: lessons from community- wide cardiovascular disease prevention programs in the US and Sweden. Scand J Public Health; 29(56):69-78.

Qiao, Y., Shin, J. I., Chen, T. K., et al. (2020). Association Between Renin-Angiotensin System Blockade Discontinuation and All-Cause Mortality Among Persons With Low Estimated Glomerular Filtration Rate. JAMA Intern Med.

Sarayani, A., Cicali, B., Henriksen, C. H., \& Brown, J. D. (2020). Safety signals for QT prolongation or Torsades de Pointes associated with azithromycin with or without chloroquine or hydroxychloroquine. Research in social \& administrative pharmacy: RSAP.

Turner, A. J., Hiscox, J. A., \& Hooper, N. M. (2004). ACE2: from vasopeptidase to SARS virus receptor. Trends Pharmacol; 25(6):291-4.

Vaduganathan, M., Vardeny, O., Michel, T., et al. (2020). Renin-Angiotensin-Aldosterone System Inhibitors in Patients with COVID-19. N Engl J Med.

Wan, Y., Shang, J., Graham, R., et al. (2020). Receptor Recognition by the Novel Coronavirus from Wuhan: an Analysis Based on Decade-Long Structural Studies of SARS Coronavirus. J Virol; 94.

Zhao, Y., Zhao, Z., Wang, Y., Zhou, Y., Ma, Y., \& Zuo, W. (2020) Single-cell RNA expression profiling of ACE2, the putative receptor of Wuhan 2019nCov. bioRxiv.

Zheng. Y. Y., Ma, Y. T, Zhang, J. Y., \& Xie, X. (2020). COVID-19 and the cardiovascular system. Nat Rev Cardiol. 